\title{
EQUIMULTIPLICITY AND HYPERPLANARITY
}

\author{
S. B. MULAY
}

\begin{abstract}
Let $R$ be an excellent regular local domain containing a field. Let $F$ be a nonzero principal ideal in $R$ contained in $m(R)$. Then the 2-codimensional equimultiple locus of $(R, F)$ is hyperplanar.
\end{abstract}

In this article our aim is to study the equimultiple locus of a hypersurface singularity in characteristic $p$. In order to explain the problem of hyperplanarity we begin with the following important observation in characteristic zero. Consider a nonzero principal ideal $F$ in the ring $R=K[[X, Z]]$ where $X$ stands for $\left(X_{1}, \ldots, X_{n}\right)$. Assume $F R=\left(Z^{d}+a_{1} Z^{d-1}+\cdots+a_{d}\right) R$ and ord $_{R} F=d$. Then for any irreducible component of the equimultiple locus defined by a prime ideal $P$ in $R$, i.e., for any $P$ with $F \in P^{(d)}$, we have $D_{Z}^{i} F \in P^{(d-i)}$, where $D_{Z}$ is the usual partial derivative with respect to $Z$. In particular, for $i=d-1$, we obtain $d ! Z+(d-1) ! a_{1}$ to be an element of $P$. Thus, dividing by $d$ ! we have $Z+a_{1} / d$ in $P$. Since $Z+a_{1} / d$ is a regular parameter of $R$, we interpret this as saying that each irreducible component of the equimultiple locus (of $F$ in $R$ ) is hyperplanar. From its expression it is evident that $Z+a_{1} / d$ does not depend on $P$. Replacing the above computation by the Tschirnhaussen transformation, Abhyanker [1] proved that if $d$ is not divisible by the characteristic of $K$ then the equimultiple locus of $F$ is hyperplanar; and he initiated the investigation in the general case.

In the main theorem of this article we establish the hyperplanarity of that part of the equimultiple locus which has codimension two in the ambient space. Obviously for a surface $F$ in three-space, this implies that the entire equimultiple locus is hyperplanar. Our proof is existential in nature and does not produce an explicit hyperplane. Another feature of this proof is that it does not depend on the characteristic.

Finally we note that R. Narasimhan [6] has given an example of a prime ideal $P$ in $K$ [ $X, Y, Z, W]](C h a r K=2)$ which defines an equimultiple curve for a three-fold $F$ and does not contain any regular parameter.

1. Preliminaries. All rings are assumed to be noetherian commutative with unity. We follow the usual notations such as $\operatorname{rad}(I)$ for the radical of an ideal $I$ in a ring $R, m(R)$ for the maximal ideal in a local ring $R$, and $P^{(d)}$ for the $d$ th symbolic power of a prime ideal $P$ in a domain $R$, etc.

Received by the editors January 3, 1983

1980 Mathematics Subject Classification. Primary 14B05.

(C)1983 American Mathematical Society $0002-9939 / 83 \$ 1.00+\$ .25$ per page 
1.1 Equimultiple locus. Let $R$ be a domain and $F$ a principal ideal in $R$. For any positive integer $d$ let $\Pi(R, F, d)=\left\{P \in \operatorname{Spec}(R) \mid F \subset P^{(d)}\right\}$, and let $\Pi^{i}(R, F, d)$ $=\left\{P \in \Pi(R, F, d) \mid \operatorname{dim} R_{P}=i\right\}$ denote the $i$-codimensional part of $\Pi(R, F, d)$. If $R$ is a regular local domain and $F$ is a nonzero principal ideal contained in $m(R)$, then let $\mathbf{E}(R, F)=\Pi(R, F$, ord $R$ and $F)$ and $\mathbf{E}^{i}(R, F)=\Pi^{i}(R, F$, ord $R)$. In this case we call $\mathbf{E}(R, F)$ the equimultiple locus of $(R, F)$ and $\mathbf{E}^{i}(R, F)$ the $i$-codimensional equimultiple locus of $(R, F)$. For any subset $E$ of $\operatorname{Spec}(R)$, by $I(E)$ we denote the intersection of all elements of $E$. Note that $I(E)$ is a radical ideal in $R$. Let $A$ be an overdomain of $R$ and $E$ a subset of $\operatorname{Spec}(R)$. We set $E_{A}=\{P \in$ $\operatorname{Spec}(A) \mid P$ is a minimal prime divisor of $Q A$ for some $Q \in E\}$.

1.2 Closedness of equimultiple locus. Let $R$ be an excellent regular domain and let $F$ be a nonzero nonunit principal ideal in $R$. Then $\Pi(R, F, d)$ is a closed subset of $\operatorname{Spec}(R)$. In particular, if $\Pi^{\mathrm{l}}(R, F, d)=\varnothing$, then $\Pi^{2}(R, F, d)$ is a finite set. (See 40.3 of [5] and 34.A of [4].)

1.3 Hyperplanarity. Let $R$ be a regular local domain. A subset $E$ of $\operatorname{Spec}(R)$ is said to be hyperplanar if there exists a regular parameter $z$ of $R$ such that $z \in P$ for all $P$ in $E$.

(a) A subset $E$ is hyperplanar iff $I(E) \not \subset M(R)^{2}$.

(b) If $E$ is hyperplanar then every subset of $E$ is hyperplanar.

(c) Let $F$ be a nonzero principal ideal contained in $M(R)$. If $\mathbf{E}^{\prime}(R, F) \neq \varnothing$, then there exists a regular parameter $z$ of $R$ such that $z^{d} R=F R$, where $d=\operatorname{ord}_{R} F$; and hence $\mathbf{E}(R, F)$ is hyperplanar.

1.4 Completion. With $R$ and $F$ as in 1.3 , let $\hat{R}$ denote the completion of $R$, and let $E$ be a subset of $\operatorname{Spec}(R)$. Then we have:

(a) If $E \subset \mathbf{E}^{i}(R, F)$, then $E_{\hat{R}} \subset \mathbf{E}^{i}(\hat{R}, F \hat{R})$ (see 22.9 of [5]). In particular if $\mathbf{E}^{i}(R, F) \neq \varnothing$ then $\mathbf{E}^{i}(\hat{R}, F \hat{R}) \neq \varnothing$;

(b) If $R$ is pseudo-geometric (in particular if $R$ is excellent) and if $E$ is a finite set then $I\left(E_{\hat{R}}\right)=I(E) \hat{R}$ (see 36.4 and 18.11 of [5]). Consequently for a finite subset $E$, hyperplanarity of $E_{\hat{R}}$ is equivalent to that of $E$.

(c) $\hat{R}$ is an excellent local domain (see 34.B of [4]).

1.5 Extension of the ground field. Let $R$ and $F$ be as in 1.3. Let $x$ be an indeterminate over $R$, and let $T$ be the set consisting of polynomials $f \in R[x]$ whose coefficients generate the unit ideal in $R$. Then $T$ is a multiplicatively closed subset of $R[x]$. By $R(x)$ we denote the ring $T^{-1} R[x]$. The facts listed below follow from (6.17) of $[5]$.

(i) $R(x)$ is a regular local domain dominating $R, m(R) R(x)=m(R(x))$ and the residue field of $R(x)$ is infinite.

(ii) For every $P \in \operatorname{Spec}(R), P R(x)$ is a prime ideal in $R(x), P R(x) \cap R=P$ and height $[P R(x)]=$ height $P$.

(iii) For ideals $I_{1}, \ldots, I_{n}$ in $R$ we have

$$
\left(I_{1} \cap \cdots \cap I_{n}\right) R(x)=I_{1} R(x) \cap \cdots \cap I_{n} R(x) .
$$

If $R$ is excellent then 34.A of [4] implies $R(x)$ is excellent.

Note that $m(R(x))^{n} \cap R=m(R)^{n}$ and if $P \in \mathbf{E}^{i}(R, F)$ then $P R(x)$ is in $\mathbf{E}^{i}(R(x) F R(x))$. Also if $E$ is a finite subset of $\operatorname{Spec}(R)$ then $I(E) R(x)=I\left(E_{R(x)}\right)$; in particular, $E$ is hyperplanar iff $E_{R(x)}$ is hyperplanar. 
2. Triodes. By a triode we mean a triple $(R, S, z)$ where $R$ and $S$ are regular local domains and $z$ is a nonzero element in $R$ such that $R$ dominates $S$ and there exists an epimorphism $u: R \rightarrow S$ with $\operatorname{ker}(u)=z R$ and $u(b)=b$ for all $b \in S$.

Note that $R$ is residually rational over $S, m(R)=z R+m(S) R$, and from known properties of regular local rings it follows that $\operatorname{dim} R=1+\operatorname{dim} S$. For a nonnegative integer $e$ we have $m(S)^{e} \subset m(R)^{e}$ and $u\left(m(R)^{e}\right)=m(S)^{e}$. Obviously ord $_{R} b \geqslant$ $\operatorname{ord}_{S} b$ for all $b$ in $S$. Conversely, if $b \in S \cap m(R)^{e}$, then $b=u(b) \in u\left(m(R)^{e}\right)=$ $m(S)^{e}$. Hence $\operatorname{ord}_{R} b=\operatorname{ord}_{S} b$, for all $b$ in $S$. The following lemma is proved in the 10 th section of [1]. We repeat it for convenience of the reader.

2.1. Assume $(R, S, z)$ is a triode. Let $f=b(0) z^{d}+b(1) z^{d-1}+\cdots+b(d)$ where $d$ is a positive integer and $b(0), \ldots, b(d)$ are in $S$. Let $\nu$ be a nonnegative integer. If $\operatorname{ord}_{S} b(j)<j(\nu+1)$ for some $j$ with $0 \leqslant j \leqslant d$, then $\operatorname{ord}_{R} f<d(\nu+1)$.

Proof. Let $e$ be the greatest integer with $0 \leqslant e \leqslant d$ such that $\operatorname{ord}_{s} b(e)<e(\nu+1)$. Let $g=b(0) z^{e}+b(1) z^{e-1}+\cdots+b(e)$. Then $\operatorname{ord}_{R} g \leqslant \operatorname{ord}_{S} u(g)=\operatorname{ord}_{S} b(e)<$ $e(\nu+1)$ and hence $\operatorname{ord}_{R} g z^{d-e}<d+e \nu$. Also ord $R\left(f-g z^{d-e}\right) \geqslant d+e \nu$. Therefore ord $\operatorname{or}_{R} f<d(1+\nu)$.

2.2. Let $(R, S, z)$ be a triode and let $Z$ be an indeterminate over $S$. Let $\psi$ : $S[Z] \rightarrow S[z]$ be the ring homomorphism defined by $\psi(Z)=z$ and $\psi(b)=b$ for all $b \in S$. Then $\psi$ is an isomorphism.

Proof. Clearly $\psi$ is an epimorphism and $\operatorname{Ker}(\psi) \cap S=0$. Let $f$ be an element of $S[Z] \backslash S$. Then $f=b(0) Z^{d}+b(1) Z^{d-1}+\cdots+b(d)$ with $b(i) \in S$ for $0 \leqslant i \leqslant d$, where $d$ is a positive integer. If $b(j) \neq 0$ for some $j$ with $1 \leqslant j \leqslant d$, we can find a nonnegative integer $\nu$ such that $\operatorname{ord}_{s} b(j)<j(\nu+1)$. In this case, from 2.1 we obtain ord $\operatorname{od}_{R} \psi(f)<d(\nu+1)$, and hence $\psi(f) \neq 0$. Assuming $f \in \operatorname{Ker}(\psi)$ we must have $b(j)=0$ for $1 \leqslant j \leqslant d$. Now $f=b(0) Z^{d}$ and $\psi(f)=b(0) z^{d}=0$. Since $z$ is a nonzero-divisor in $R$ we conclude that $b(0)=0$.

2.3. Let $(R, S, z)$ be a triode. If $\hat{R}$ and $\hat{S}$ denote the completions of $R$ and $S$, respectively, then $(\hat{R}, \hat{S}, z)$ is a triode and $\hat{R}=\hat{S}[[z]]$.

Proof. Clearly $(\hat{R}, \hat{S}, z)$ is a triode. Let $Z$ be indeterminate over $\hat{S}$. Since $\hat{R}$ is complete and $z \in m(\hat{R})$, there exists a homomorphism $V: \hat{S}[[Z]] \rightarrow \hat{R}$ such that $V(Z)=z$ and $V(b)=b$ for all $b \in \hat{S}$. Because $m(\hat{R})=z \hat{R}+m(\hat{S}) \hat{R}$ and $\hat{R}$ is residually rational over $\hat{S}, V$ is an epimorphism. Now $\operatorname{dim} \hat{S}[[Z]]=\operatorname{dim} \hat{S}+1=$ $\operatorname{dim} \hat{R}$ and hence $V$ must be injective.

2.4. Weierstrass Preparation Theorem. Let $(R, S, z)$ be a triode. Assume that $R$ and $S$ are complete. Let $F$ be a principal ideal in $R$. If $F \not \subset m(S) R$, then there exists $a$ distinguished polynomial $f(Z) \in S[Z]$ (where $Z$ is an indeterminate over $S$ ) such that $f(z) R=F R$.

Proof. Follows from 2.3 and [2,(10.2)].

2.5. Let $(R, S, z)$ be a triode such that $S$ is an excellent local ring. Let $F$ be a principal ideal in $S[z]$ and let $d$ be a positive integer. Assume $\Pi^{i}(R, F R, d) \neq \varnothing$. If $P \in \Pi^{i}(R, F R, d)$ then $P \cap S[z]$ is in $\Pi(S[z], F, d)$ and height $(P \cap S[z]) \leqslant i$. 
Proof. Let $Q=P \cap S[z]$. Then $Q$ is a nonzero prime ideal in $S[z]$ and $Q$ is contained in $M(R) \cap S[z]$. Let $M=m(R) \cap S[z]=m(S) S[z]+z S[z]$ and $A=$ $S[z]_{M}$. It can be easily seen that $(A, S, z)$ is a triode. Let $\hat{R}, \hat{A}$ and $\hat{S}$ denote the completions of $R, A$ and $S$, respectively. From 2.3 it follows that $\hat{R}=\hat{S}[[z]]=\hat{A}$. Let $P^{*}$ be a prime ideal in $\hat{R}$ with $P^{*} \cap R=P$. Clearly $R$ dominates $A$, and $P^{*} \cap A=Q A$. Since there exist $f \in S[z]$ and $u \in R \backslash P$ with $F=f S[z]$ and $u f \in P^{d}$, we have $u f \in\left(P^{*}\right)^{d}$. Obviously $u \notin P^{*}$. Hence $P^{*} \in \Pi(\hat{R}, F \hat{R}, d)$. Let $B=\hat{A}_{P^{*}}$ and $C=A_{Q A}$. Then $B, C$ are regular local domains, $B$ dominates $C$, and $\operatorname{ord}_{B} f \geqslant d$. Because $S$ is an excellent local ring, $A$ also is an excellent local ring. Consequently, $B / m(C) B$ is a regular local domain and $\operatorname{dim} B=\operatorname{dim} C+\operatorname{dim}[B / m(C) B]$. (See 33.A of [4].)

Now it is easy to see that $\operatorname{ord}_{C} f \geqslant d$. In conclusion we have $F \subset Q^{(d)}$, i.e., $Q \in \Pi(S[z], F, d)$. By 22.9 of [5], we have height $Q=\operatorname{height}(Q \hat{A}) \leqslant \operatorname{height}(P \hat{R})=$ height $P$.

2.6. Let $n$ and $d$ be positive integers. Let $R$ be a regular local domain of dimension $n$ containing an infinite field. Let $F$ be a nonzero principal ideal in $R$ contained in $m(R)^{d}$. Assume that $F$ is not contained in $m(R)^{d+1}$. Then there exists a triode $(\hat{R}, S, z)$ with $f(Z) \in S[Z]$ such that $\hat{R}$ is the completion of $R, f(Z)$ is a monic polynomial of degree $d$ and $f(z) \hat{R}=F \hat{R}$.

Proof. From well-known properties of completion it follows that $F \hat{R} \subset m(\hat{R})^{d}$ and $F \hat{R} \not \subset m(\hat{R})^{d+1}$. According to (4.13) of [3], there exists a basis $\left(X_{1}, \ldots, X_{n}\right)$ of $m(\hat{R})$ such that $F \hat{R} \not \subset\left(X_{1}^{d+1}, X_{2}, \ldots, X_{n}\right) \hat{R}$. By Cohen's structure theorem $\hat{R}=$ $K\left[\left[X_{1}, \ldots, X_{n}\right]\right]$, where $K$ is the coefficient field of $R$. Let $S=K\left[\left[X_{2}, \ldots, X_{n}\right]\right]$ and $z=X_{1}$. Then $F \hat{R} \not \subset m(S) \hat{R}, S$ is complete and $(\hat{R}, S, z)$ is a triode. The rest of the proof follows from 2.4 .

3. Tables. By a table we mean a five-tuple $(B, Z, A, d, f(Z))$ where $B$ is a domain, $Z$ is an indeterminate over $B, A=B[Z], d$ is a positive integer, and $f(Z) \in A$ has the form $f(Z)=Z^{d}+b(1) Z^{d-1}+\cdots+b(d)$ with $b(i) \in B$ for $1 \leqslant i \leqslant d$.

3.1. Let $(B, Z, A, d, f(Z))$ be a table. Let $P \in \operatorname{Spec}(A)$ be such that $f(Z)$ is in $P$. Let $Q=P \cap B$. Then:

(i) height $P=1+$ height $Q$.

(ii) $Q A$ is a prime ideal in $A$.

(iii) If $Z-\theta$ belongs to $P$ for some $\theta \in B$, then $P=(Z-\theta) A+Q A$.

Proof. Follows from the well-known properties of polynomial rings.

3.2. Let $\left(B^{*}, Z, A^{*}, d, f(Z)\right)$ be a table. Let $z=Z-\theta$, where $\theta \in B^{*}$ and let $P$ be a prime ideal in $A^{*}$ containing $z$. Let $Q=P \cap B^{*}$. Assume that $B_{Q}^{*}$ is a regular local domain. Then $\left(A_{P}^{*}, B_{Q}^{*}, z\right)$ is a triode.

Proof. Observe that $A^{*}=B^{*}[z], z A^{*}$ is a prime ideal in $A^{*}$, and $A_{P}^{*}$ dominates $B_{Q}^{*}$. From 3.1 it follows that $\operatorname{dim} A_{P}^{*}=1+\operatorname{dim} B_{Q}^{*}, m\left(A_{P}^{*}\right)=z A_{P}^{*}+m\left(B_{Q}^{*}\right) A_{P}^{*}$, and $z A_{P}^{*} \cap B_{Q}^{*}=0$. Hence $A_{P}^{*}$ is a regular local domain. Let $\sigma: A_{P}^{*} / z A_{P}^{*} \rightarrow B_{Q}^{*}$ be the canonical isomorphism, $h: A_{P}^{*} \rightarrow A_{P}^{*} / z A_{P}^{*}$ be the canonical epimorphism and $u=$ $\sigma \circ h$. Then $u$ is an epimorphism, $\operatorname{Ker}(u)=z A_{P}^{*}$ and $u(b)=b$ for all $b \in B_{Q}^{*}$. 
To avoid repetition we fix a table $(B, Z, A, d, f(Z))$, without an explicit mention of it, in the rest of this section.

3.3. Let $P \in \Pi(A, f(Z) A, d), Q=P \cap B$ and $\alpha$ be an element of $B$. Assume that $B_{Q}$ is a regular local domain. Let $\theta$ be an element of $B_{Q}$ with $\alpha \theta$ in $B$. If $\alpha(Z-\theta) \in P$, then $f(\theta) \alpha^{d}$ is in $B \cap Q^{(d)}$.

Proof. If $\alpha \in Q$ we have nothing to prove. Assume $\alpha \notin Q$. Let $T=B \backslash Q$, $A^{*}=T^{-1} A, B^{*}=B_{Q}=T^{-1} B$. Then $(Z-\theta) \in P A^{*}$ and $\left(B^{*}, Z, A^{*}, d, f(Z)\right)$ is a table. Obviously $A_{P}=A_{P^{*}}^{*}$ where $P^{*}=P A^{*}$. From 3.1 it follows that $\left(A_{P}, B_{Q}, z\right)$ is a triode where $z=Z-\theta$. As an element of $A_{P}, f(Z)$ has the form $z^{d}+C_{1} z^{d-1}$ $+\cdots+C_{d}$, where $C_{i} \in B_{Q}$ for $1 \leqslant i \leqslant d$ and $C_{d}=f(\theta)$. Now the assertion follows from 2.1 .

3.4. Assume $\Pi(A, f(Z) A, d) \neq \varnothing$. Let $P \in \Pi(A, f(Z) A, d)$ and $Q=P \cap B$. Then:

(i) there exists $\eta \in B \backslash Q, \psi \in B(\eta, \psi$ depending on $P)$ such that $\eta Z+\psi \in P$;

(ii) $\operatorname{rad}(Q A+f(Z) A)=P$.

Proof. Note that $Q \in \operatorname{Spec}(B), Q A \in \operatorname{Spec}(A)$ and $f(Z) \notin Q A$. Let $h: A \rightarrow$ $A / Q A$ be the canonical epimorphism, $R=h(A), S=h(B), \bar{Z}=h(Z), f(\bar{Z})=$ $h(f(Z))$ and $\bar{P}=h(P)$. Then $(S, \bar{Z}, R, d, f(\bar{Z}))$ is a table, $0 \neq f(\bar{Z}) \in \bar{P}$ and $\bar{P} \in \Pi(R, f(\bar{Z}) R, d)$. Let $T=S \backslash\{0\}, R_{0}=T^{-1} R \supset R, k=T^{-1} S \subset R_{0}$ and $P_{0}=$ $\bar{P} R_{0}$. Then $\left(k, \bar{Z}, R_{0}, d, f(\bar{Z})\right)$ is a table, $k$ is a field and $P_{0} \in \Pi\left(R_{0}, f(\bar{Z}) R_{0}, d\right)$.

(i) Clearly $0 \neq P_{0}$ is a principal prime ideal in $R_{0}=k[\bar{Z}]$. Let $g(\bar{Z})$ be the monic generator of $P$. Then $f(\bar{Z})=c g(\bar{Z})^{d}$ for some $c \in R_{0}$. It follows that $c=1$ and $g(\bar{Z})=\bar{Z}+\theta$ for some $\theta \in k$. Let $\beta \in T$ be such that $\beta \theta \in S$. Then $\beta \bar{Z}+\beta \theta=$ $\beta(\bar{Z}+\theta) \in P_{0} \cap R=\bar{P}$. Let $\eta, \psi \in B$ be such that $h(\eta)=\beta$ and $h(\psi)=\beta \theta$. Now $h(\eta Z+\psi)=\beta \bar{Z}+\beta \theta \in \bar{P}=h(P)$, hence $\eta Z+\psi \in P$. Also $h(\eta)=\beta \in T$ implies that $\eta \notin Q$.

(ii) Let $J=h(Q A+f(Z) A)$. Then $J=f(\bar{Z}) R \subset \bar{P}$. It is easy to see that if $P^{\prime}$ is any minimal prime of $J$ then height $P^{\prime}=1$ and $P^{\prime} \cap S=0$. (See 3.1.) Consequently, if $P_{1}=P^{\prime} R_{0}$, then $P_{1} \neq R_{0}$. Now we have $f(\bar{Z}) R_{0} \subset P_{1}$, i.e., $(\bar{Z}+\theta)^{d} R_{0} \subset P_{1}$ and hence $\bar{Z}+\theta \in P_{1}$. It follows that $P_{0}=(\bar{Z}+\theta) R_{0} \subset P_{1}$ and $\bar{P}=P_{0} \cap R \subset P_{1} \cap R$ $=P^{\prime}$. In conclusion we have $\bar{P}=\operatorname{rad}(J)$. The rest of the proof is straightforward.

3.5. Let $P_{1}, P_{2} \in \Pi(A, f(Z) A, d), Q_{1}=P_{1} \cap B$ and $Q_{2}=P_{2} \cap B$. If $Q_{1} \subset Q_{2}$ then $P_{1} \subset P_{2}$.

Proof. Follows from 3.4(ii).

3.6 Definition. For an ideal $J$ in $A$ we define $\Delta(J)=\{\alpha \in B \mid \alpha Z+\beta \in J$ for some $\beta \in B\}$.

Note that $\Delta(J)$ is an ideal in $B, \Delta(J)=B$ iff there exists $t \in B$ with $Z+t \in J$.

3.7. Let $P \in \Pi(A, f(Z) A, d)$ and $Q=P \cap B$. Then $\Delta(P) \not \subset Q$. Furthermore, if $\eta \in B \backslash Q, \psi \in B$ are such that $\eta Z+\psi \in P$, then we have $\Delta(P)=[(\eta B+Q B): \psi B]$.

Proof. $\Delta(P) \not \subset Q$ follows from 3.4. 
Let $\eta \in B \backslash Q, \psi \in B$ be such that $\eta Z+\psi \in P$. For $\alpha \in[(\eta B+Q B): \psi B]$, $\alpha \psi=\beta \eta+q$ where $\beta \in B$ and $q \in Q \subset P$. Since $\eta Z+\psi \in P, q \in P$; it follows that $\alpha(\eta Z+\psi)-q=\alpha \eta Z+\beta \eta=\eta(\alpha Z+\beta) \in P$. But $\eta \notin P$ and hence $\alpha Z+\beta \in P$. Consequently $[(\eta B+Q B): \psi B] \subset \Delta(P)$. Conversely, if $\alpha \in \Delta(P)$ then for some $\beta \in B$, both $\alpha Z+\beta$ and $\alpha(\eta Z+\psi)$ are in $P$. Hence $\alpha(\eta Z+\psi)-$ $\eta(\alpha Z+\beta)=\alpha \psi-\eta \beta$ is in $P$. Now $\alpha \psi-\eta \beta \in B$ implies $\alpha \psi-\eta \beta \in P \cap B=Q$. It follows that $\alpha \in[(\eta B+Q B): \psi B]$, from which $\Delta(P)=[(\eta B+Q B): \psi B]$ follows.

3.8. For $1 \leqslant i \leqslant n$ let $P_{i} \in \Pi(A, f(Z) A, d)$ be such that $P_{i} \not \subset P_{j}$ for $i \neq j, 1 \leqslant i \leqslant \eta$, $1 \leqslant j \leqslant n$. Let $J=P_{1} \cap \cdots \cap P_{n}, I=J \cap B$ and $T=Q_{1} \cup \cdots \cup Q_{n}$, where $Q_{i}=P_{i}$ $\cap B$ for $1 \leqslant i \leqslant n$. Then $\Delta(J) \not \subset T$. Furthermore, if $\eta \in B \backslash T, \psi \in B$ are such that $\eta Z+\psi \in J$, then we have $\Delta(J)=[(\eta B+I B): \psi B]$.

Proof. The case $n=1$ is the statement of 3.7.

Assume $n \geqslant 2$. From 3.7 it follows that there exist elements $\eta_{i} \in B \backslash Q_{i}, \quad \psi_{i} \in B$ for $1 \leqslant i \leqslant n$ such that $\eta_{i} Z+\psi_{i} \in P_{i}$ for $1 \leqslant i \leqslant n$. From 3.5 we deduce that $Q_{i} \not \subset Q_{j}$ for $i \neq j, 1 \leqslant i \leqslant n, 1 \leqslant j \leqslant n$; combining this with the fact that $Q_{i} \in$ $\operatorname{Spec}(B)$ for $1 \leqslant i \leqslant n$, we have $\left[\cap_{i \neq j} Q_{i}\right]=N(j) \not \subset Q_{j}$ for $1 \leqslant j \leqslant n$.

Let $a(j) \in N(j) \backslash Q_{j}$ for $1 \leqslant j \leqslant n$. Consider the element $\sigma=\sum a(j)\left(n_{j} Z+\psi_{j}\right)$. Clearly $\sigma \in J$. Let $\alpha=\Sigma a(j) \eta_{j}$ and $\beta=\Sigma a(j) \psi_{j}$. Then $\alpha \in B \backslash T, \psi \in B$ and $\sigma=\alpha Z+\beta \in J$. Hence $\Delta(J) \not \subset T$.

Let $\eta \in B \backslash T, \psi \in B$ be such that $\eta Z+\psi \in J$ and let $\alpha \in \Delta(J)$. Now there exists $\beta \in B$ such that $\alpha Z+\beta \in J$. Consequently $\alpha(\eta Z+\psi)-\eta(\alpha Z+\beta) \in J$, i.e., $\alpha \psi$ $-\eta \beta \in J$. Since $\alpha \psi-\eta \beta \in B$ we have $\alpha \psi-\eta \beta \in I$, i.e., $\alpha \in[(\eta B+I B): \psi B]$. Conversely for $\alpha \in[(\eta B+I B): \psi B]$ we have $\alpha \psi=\eta \beta+a$ for some $\beta \in B, a \in I$. Because $I \subset J$ we have $\alpha(\eta Z+\psi)-a=\eta(\alpha Z+\beta) \in J$. Now $\eta \notin T$ implies $\eta \notin P_{i}$ for $1 \leqslant i \leqslant n$. Hence $\alpha Z+\beta \in J$, i.e., $\alpha \in \Delta(J)$.

3.9. Assume that $B$ is a regular domain and $\Pi^{2}(A, f(Z) A, d) \neq \varnothing$. For $1 \leqslant i \leqslant n$ let $P_{i} \in \Pi^{2}(A, f(Z) A, d)$ and let $J=P_{1} \cap \cdots \cap P_{n}$. Then $\Delta(J)=B$.

Proof. Assume, if possible, that $\Delta(J) \neq B$. Let $P \in \operatorname{Spec}(B)$ be a minimal prime of $\Delta(J)$. Let $I=J \cap B$ and $Q_{i}=P_{i} \cap B$ for $1 \leqslant i \leqslant n$. Then $I=Q_{1} \cap \cdots \cap Q_{n}$, height $Q_{i}=1$ for $1 \leqslant i \leqslant n$ (see 3.1) and, hence, height $I=1$.

Clearly we have $I \subset P \not \subset T$, where $T=Q_{1} \cup \cdots \cup Q_{n}$. Let $R=B_{P}$. It follows that $R$ is a regular local domain of dimension $\geqslant 2, \Delta(J) R$ is primary for $m(R)$ and since $R$ is a UFD, IR is a nonzero principal ideal in $R$. Since $P \not \subset T$, and $P \not \subset P^{(2)}$ we can choose an $x$ in $P$ such that $x \notin T \cup P^{(2)}$. Then $x$ is a regular parameter of $R$ and hence $R / x R$ is a regular local domain of dimension $\geqslant 1$. Let $e$ be the least positive integer such that $x^{e} \in \Delta(J) R$. Choose $t \in B \backslash P$ so that $t x^{e} \in \Delta(J) B$. Let $y=t x^{e}$ and let $\beta$ be an element of $B$ with $y Z+\beta$ in $J$. Let $f(Z)=Z^{d}+c_{1} Z^{d-1}$ $+\cdots+c_{d}$, where $c_{i} \in B$ for $1 \leqslant i \leqslant d$. From 3.3 we have $\beta^{d}+c_{1} y \beta^{d-1}$ $+\cdots+c_{d} y^{d}$ in $Q_{i}^{(a)}$ for $1 \leqslant i \leqslant n$. Clearly $\beta^{d}+c_{1} y \beta^{d-1}+\cdots+c_{d} y^{d}$ belongs to $Q_{i}^{(d)} R$ for $1 \leqslant i \leqslant n$. Since $Q_{i} R$ is a principal ideal we have $Q_{i}^{(d)} R=\left(Q_{i} R\right)^{d}$ for 
$1 \leqslant i \leqslant n$. Furthermore $I^{d}=\left(Q_{1} R\right)^{d} \cap \cdots \cap\left(Q_{n} R\right)^{d}$. Let $g \in R$ be such that $g R=$ $I R$. Then for some $\lambda \in R$ we have

$$
\beta^{d}+c_{1} y \beta^{d-1}+\cdots+c_{d} y^{d}=\lambda g^{d} .
$$

Let $h: R \rightarrow R / x R$ be the canonical epimorphism. As a consequence of $(*)$ we get

$$
h(\beta)^{d}=h(\lambda) \cdot h(g)^{d} .
$$

Earlier we observed that $R / x R$ is a regular local domain, in particular, it is a normal domain. Hence $h(\beta) \in h(g R)=h(I R)$ and in turn $\beta$ is in $x R+I R$. It follows that for some $v \in B$ and for some $u \in B \backslash P, u \beta-v x$ is in $I B$. Recall that $(y Z+\beta) \in J$. Thus, we have $(u y Z+v x)$ in $J$, i.e.,

$$
\left[\left(\tau x^{e}\right) Z+v x\right] \in J \quad \text { with } x \notin T \text { and } \tau=u t \in B \backslash P .
$$

Now from our choice of $e$ and from (**) we deduce that

$$
\left[\left(\tau x^{e-1}\right) Z+v\right] \in J \quad \text { where } \tau \in B \backslash P .
$$

Consequently $\tau x^{e-1} \in \Delta(J)$, i.e., $x^{e-1} \in \Delta(J) R$. Minimality of $e$ implies $e-1=0$. Hence $\Delta(J) R=R$, but this is a contradiction.

\section{Main Theorem.}

Let $R$ be an excellent regular local domain containing a field. Let $F$ be a nonzero principal ideal in $R$, contained in $m(R)$. Then the 2-codimensional equimultiple locus of $(R, F)$ is hyperplanar.

Proof. If $\mathbf{E}^{\mathrm{l}}(R, F) \neq \varnothing$ then $\mathbf{E}(R, F)$ is hyperplanar. Hence assume $\mathbf{E}^{\mathrm{l}}(R, F)$ $=\varnothing$. In view of $1.2-1.5$, we will restrict ourselves to a complete regular local domain $R$ which contains an infinite field.

From 2.6 we deduce the existence of a triode $(R, S, z)$ and a monic polynomial $f(Z) \in S[Z]$ (where $Z$ is an indeterminate over $S$ ) of degree $d$ such that $f(z) R=F R$ and $d=\operatorname{ord}_{R} F$. Note that $S$ is a complete regular local domain and therefore $S$, $S[z]$ and $S[Z]$ are all excellent domains.

From 1.2, 2.2 and 3.9 it follows that there exists $t \in S$ with $(z+t) \in P$ for all $P \in \Pi^{2}(S[z], f(z) S[z], d)$. The rest of the proof is a simple consequence of 2.5 .

COROllary. In the above set-up, if $\operatorname{dim} R=3$ then $\mathbf{E}(R, F)$ is hyperplanar.

\section{REFERENCES}

1. S. Abhyankar, Good points of a hypersurface, preprint.

2. L_ Local analytic geometry, Academic Press, New York, 1964.

3. __ Resolution of singularities of embedded algebraic surfaces, Academic Press, New York, 1966.

4. H. Matsumura, Commutative algebra, Benjamin/Cummings, Reading, Mass., 1980.

5. M. Nagata, Local rings, Krieger, Huntington, N. Y., 1975.

6. R. Narasimhan, Hyperplanarity of the equimultiple locus, Proc. Amer. Math. Soc. 87 (1983), 403-408.

Department of Mathematics, University of Tennessee, KNoxville, Tenץ'essee 37996-1300 